\title{
Why is a noble metal catalytically active? The role of the O-Ag interaction in the function of silver as an oxidation catalyst
}

\author{
Wei-Xue $\mathrm{Li}^{1}$, Catherine Stampfl${ }^{1,2}$, and Matthias Scheffler ${ }^{1}$ \\ ${ }^{1}$ Fritz-Haber-Institut der Max-Planck-Gesellschaft, \\ Faradayweg 4-6, D-14195 Berlin-Dahlem, Germany \\ ${ }^{2}$ Department of Physics and Astronomy, Northwestern University, Evanston, Il. 60208
}

(Dated: Received 12 August 2002)

\begin{abstract}
Extensive density-functional theory calculations, and taking into account temperature and pressure, affords a comprehensive picture of the behavior and interaction of oxygen and $\operatorname{Ag}(111)$, and provides valuable insight into the function of silver as an oxidation catalyst. The obtained phasediagram reveals the most stable species present in a given environment and thus identifies (and excludes) possibly active oxygen species. In particular, for the conditions of ethylene epoxidation, a thin oxide-like structure is most stable, suggesting that such atomic O species are actuating the catalysis, in contrast to hitherto proposed molecular-like species. Copyright (2003) by The Americal Physical Society.

PACS numbers: PACS numbers: 81.65.Mq, 68.43.Bc, 82.65.My
\end{abstract}

The importance of reactant bond strength in heterogeneous catalysis is demonstrated by the so-called "volcano curves"1,2.3 which relate the reaction rate to the adsorption energy of the reaction intermediates. Typically a maximum occurs in the rate at moderate values. This behavior underlies the expectation that a good catalyst should readily dissociate adparticles but not bind the fragments too strongly - a concept put forward by Sabatier in the early 1900's, which recent theoretical and experimental studies have re-addressed $\frac{4.5}{*}$ Despite this expectation, silver, as a noble metal, which binds adparticles only relatively weakly on the surface, is a very important heterogeneous catalyst for various oxidation reactions. For example, partial oxidation of methanol to formaldehyde, as carried out at atmospheric pressure and temperatures of $800-900 \mathrm{~K}, \frac{6,7}{, 2}$ as well as the selective oxidation of ethylene to epoxide, also conducted at atmospheric pressure and at temperatures of 500-600 K $\frac{8}{2}$

Although numerous investigations aimed at elucidating the active oxygen species involved in the abovementioned reactions have been carried out, it can be said that presently there is considerable confusion, and no unambiguous identifications have been made. For example, unclear issues concern the debate of molecular (ozone-like) $)^{\underline{\underline{y}}}$ versus atomic $\underline{\underline{10}}$ oxygen as the active species for ethylene epoxidation, the proposed importance of "bulk-dissolved" oxygen and a "strongly bound, surface-embedded" species (which desorbs at $900 \mathrm{~K}$ ) for the partial oxidation of methanol, $\frac{6}{a}$ as well as the possible role of chemisorbed or surface oxide-like species. Part of the reason for this lack of understanding is due to the difficulties associated with the pressure and materials gaps which exist for the oxygen-silver system. That is, the behavior is different under ultra-high vacuum (UHV) conditions, where the system can be analysed quantitatively, to that under the high temperature and pressure conditions of catalysis, where it is much more difficult to obtain the same level of microscopic information.

Our theoretical strategy, aimed at gaining an under- standing of the function of silver as an oxidation catalyst, in spite of its relatively weak binding capability, and to identify (and exclude) possibly active species, is to carry out extensive and systematic density functional theory (DFT) calculations for all conceivably relevant oxygen species. We take the effect of temperature $(T)$ and pressure $(p)$ into account through the oxygen chemical potential, and thus determine the $(T, p)$ phase-diagram which describes the surface phases from UHV right up to the conditions of real catalysis. We study oxygen at the (111) surface of silver as experiments indicate that this orientation is an important crystal face for real silver catalysts in that at high temperatures, facets with this face result $\stackrel{6.11}{ }$ Our general findings, however, are expected to be relevant for silver per se, and possibly to have implications for gold, also a noble metal oxidation catalyst 12 which, for the (111) surface, exhibits a restructuring and chemisorption of oxygen atoms at elevated temperatures

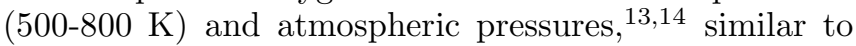
$\mathrm{Ag}(111)$. They could also have consequences for copper, which catalyses the oxidation of methanol to formaldehyde 15

Our calculations employ the pseudopotential16.17 plane-wave method ${ }^{18}$ with the generalized gradient approximation (GGA) $\frac{19}{19}$ for the exchange-correlation functional. Five Ag layers are used in the supercell to model the $\mathrm{O} / \mathrm{Ag}$ surfaces, which are created on one side of the slab, with a vacuum region of $15 \AA$. The same k-point sampling is used for all structures. It corresponds to 21 points in the irreducible part of the Brillouin zone of the $(1 \times 1) \mathrm{Ag}(111)$ surface cell. An energy cutoff of 50 Ry is used and full relaxation of the top two or three silver layers, and the $\mathrm{O}$ atoms, is taken into account. We include the spin-polarization energy in calculation of the total energy of the free atoms and molecules. Further details are reported in Ref. 20 .

The oxygen species investigated, for a wide range of coverages, include: on-surface sites, surface- and bulksubstitutional sites, interstitial sites under the surface $\mathrm{Ag}$ 


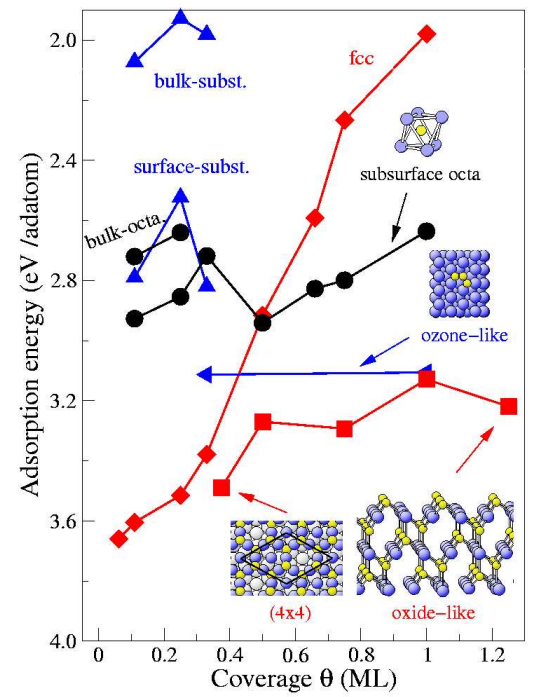

FIG. 1: Selected results of average adsorption energies (with respect to atomic $\mathrm{O}$ ) versus coverage for various structures: pure on-surface oxygen in fcc-hollow sites (red diamonds), surface- and bulk-substitutional adsorption (blue triangles), pure sub-surface oxygen in octahedral sites under the first ("subsurface-octa.") and second ("bulk-octa.") Ag layers (black circles), an $\mathrm{O}_{3}$-like molecule adsorbed at a surface $\mathrm{Ag}$ vacancy (left-pointing black triangles), and oxide-like structures (red squares). Oxygen atoms are depicted as yellow spheres and Ag atoms as the larger blue (and grey) spheres.

layer and deeper in the bulk, as well as oxide-like structures, and a molecular ozone-like species adsorbed at a surface $\mathrm{Ag}$ vacancy.

The main important adsorption energies are summarized in Fig. 1, where for each "type" of oxygen atom, only the lowest energy structure is shown. For on-surface adsorption, the fcc-hollow site is preferred for all coverages investigated. For sub-surface adsorption, the octa. site under the first Ag layer is the most favorable. This is also the case for adsorption under the second $\mathrm{Ag}$ layer which may be taken to represent "bulk dissolved" oxygen. For all sub-surface sites, adsorption under the second layer is less favorable than under the first layer. In view of the unfavorable energy of oxygen in the bulk, high concentrations of bulk-dissolved oxygen in the perfect fcc lattice of silver are improbable.

Surface- or bulk-substitutional adsorption is seen from Fig. 1 to be unfavorable, as is the ozone-like species. To investigate the formation of oxide-like structures, we performed systematic investigations for geometries involving coverages of $0.50,0.75,1.0$, and $1.25 \mathrm{ML}$ using $(2 \times 2)$ cells. In particular, we tested combinations of on-surface fcc and hcp sites, and the three possible sub-surface sites under the first Ag layer. The atomic geometry of the favored structure consists of $0.25 \mathrm{ML}$ of oxygen on the surface and 0.5 ML in between the Ag layers. For increasing O concentrations, this structure "grows" by filling deeper lying sites between the Ag layers. The structure for a total coverage of $1.25 \mathrm{ML}$ oxygen is sketched in the inset

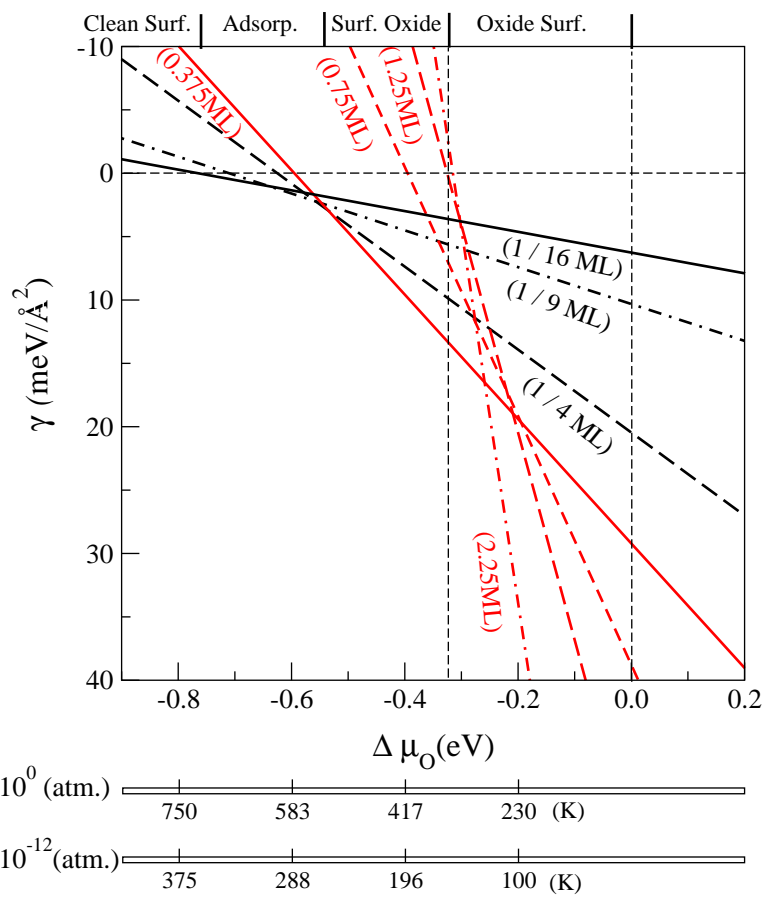

FIG. 2: Surface free energies for various low energy structures as a function of the $\mathrm{O}$ chemical potential where $\Delta \mu_{\mathrm{O}}=$ $\mu_{\mathrm{O}}-\frac{1}{2} E_{\mathrm{O}_{2}}^{\text {total }}$. For pressures of $10^{-12} \mathrm{~atm}$. (UHV) and $1 \mathrm{~atm}$. (condition of catalysis), the corresponding temperatures are given. The temperature labels correspond to the tick marks and labels for the chemical potential. The labels "0.75 ML", "1.25 ML", and "2.25 ML", indicate the O-concentrations in the corresponding oxide-like structures. Accordingly the $(4 \times 4)$ structure has the label $0.375 \mathrm{ML}$, and the adsorption structures $1 / 4,1 / 9$, and $1 / 16 \mathrm{ML}$. At the top of the figure, the "material type" which is stable in the corresponding range of chemical potential are listed and indicated by the colored regions.

of Fig. 1. In addition, we carried out calculations for the recently proposed $(4 \times 4)$ thin "surface-oxide" structure 21 which involves an $\mathrm{O}-\mathrm{Ag}-\mathrm{O}$ bonding unit (and electronic structure) similar to the upper tri-layer of the oxide structures described above (see insets of Fig. 1). The average adsorption energies of all these oxide-like structures are displayed in Fig. 1, shown as red squares. Their atomic geometries are in fact very similar to the (111) surface of $\mathrm{Ag}_{2} \mathrm{O}$.

We turn now to investigate the effect of pressure and temperature on the stability of the various structures. To do this we calculate the surface free energy,

$$
\gamma(T, p)=\left(G-N_{\mathrm{Ag}} \mu_{\mathrm{Ag}}-N_{\mathrm{O}} \mu_{\mathrm{O}}\right) / A,
$$

where $G=G_{\mathrm{O} / \operatorname{Ag}(111)}^{\mathrm{slab}}-G_{\mathrm{Ag}(111)}^{\mathrm{slab}}$ and the first and second terms on the right-hand-side are the free energies of the $\mathrm{O} / \mathrm{Ag}$ surface under consideration and that of the reference system, i.e. the clean $\operatorname{Ag}(111)$ slab, respectively. $A$ is surface area in $\AA^{2}$ and $N_{\mathrm{O}}$ is the number of oxygen atoms. $N_{\mathrm{Ag}}$ is the number of silver atoms (with respect to the reference system) and $\mu_{\mathrm{Ag}}$ is the $\mathrm{Ag}$ chemical potential, which is the free energy of an $\mathrm{Ag}$ atom 
in bulk fcc silver. (Consideration of $N_{\mathrm{Ag}}$ and $\mu_{\mathrm{Ag}}$ are necessary only for the substitutional structures and the $(4 \times 4)$ phase). The $T$ and $p$ dependence is given by $\mu_{\mathrm{O}}$, the oxygen chemical potential,,$^{24}$

$\mu_{\mathrm{O}}(T, p)=1 / 2\left[E_{\mathrm{O}_{2}}^{\mathrm{total}}+\tilde{\mu}_{\mathrm{O}_{2}}\left(T, p^{0}\right)+k_{B} T \ln \left(\frac{p_{\mathrm{O}_{2}}}{p^{0}}\right)\right]$,

where $p^{0}$ corresponds to atmospheric pressure and $\tilde{\mu}_{\mathrm{O}_{2}}\left(T, p^{0}\right)$ includes the contribution from rotations and vibrations of the molecule, as well as the ideal-gas entropy at 1 atmosphere. Here we use experimental values from thermodynamic tables.

Through consideration of the difference in the vibrational and entropic contributions of $G_{\mathrm{O} / \mathrm{Ag}(111)}^{\mathrm{slab}}$ and $G_{\mathrm{Ag}(111)}^{\text {slab }}$, we find that it can be neglectedi ${ }^{22}$ Thus, for evaluating the difference of the two $G^{\text {slab }}$ values, we replace $G^{\text {slab }}$ by the total energy. We choose the zero of the $\mathrm{O}$ chemical potential to be half the total energy of $\mathrm{O}_{2}$ calculated as $1 / 2 E_{\mathrm{O}_{2}}^{\text {total }} \simeq E_{\mathrm{Ag}_{2} \mathrm{O}}^{\text {total }}-2 E_{\mathrm{Ag}}^{\text {total }}-H_{\mathrm{Ag}_{2} \mathrm{O}-\text { bulk }}^{f}$. That is, as the energy difference between bulk silver oxide $\left(E_{\mathrm{Ag}_{2} \mathrm{O}}^{\text {total }}\right)$ and bulk silver $\left(2 E_{\mathrm{Ag}}^{\text {total }}\right)$, and the experimental value of the heat of formation $\left(H_{\mathrm{Ag}_{2} \mathrm{O}-\text { bulk }}^{f}\right)$ which is $-0.323 \mathrm{eV}^{23}$

The obtained results are displayed in Fig. 2. The range of $\Delta \mu_{\mathrm{O}}$ (defined as $\mu_{\mathrm{O}}-\frac{1}{2} E_{\mathrm{O}_{2}}^{\text {total }}$ ) between the vertical dashed lines corresponds to the heat of formation of $\mathrm{Ag}_{2} \mathrm{O}$, i.e., to the range in which bulk silver oxide is stable. It can be seen that for more O-rich conditions (right) the thicker oxide-like structures are favored over chemisorption on the surface. Clearly, in the limit of an infinitely thick film, the corresponding line would be very close to the vertical one at $\Delta \mu_{\mathrm{O}}=-0.323 \mathrm{eV}$. It can be obtained from Fig. 2 that bulk silver oxide is stable to only around $350 \mathrm{~K}$ at atmospheric pressure, which is less than the experimental value of $460 \mathrm{~K}$. At $460 \mathrm{~K}$, the bulk oxide is unstable when the pressure is lower than $10^{3} \mathrm{~atm}$. i.e. compared to $1 \mathrm{~atm}$. as observed experimentally. This difference may be due to systematic errors of the DFT approach ${ }^{25}$ and/or to neglect of the entropy contributions. Therefore in Fig. 2 (and Fig. 3), we generally expect that the temperature is underestimated, but the error is less than $110 \mathrm{~K}$, while correspondingly, the pressure is overestimated. For the temperature values quoted below, we give an estimate of the upper limit in brackets, that is, $110 \mathrm{~K}$ higher than that obtained from Fig. 2. In spite of these uncertainties, we believe that the relative stability of the various systems and our general understanding and conclusions will not be affected.

Due to the above-mentioned low thermal stability of silver oxide and the thicker oxide-like structures, our results can safely rule them out as playing an important role in the catalytic reactions. For values of the $\mathrm{O}$ chemical potential further to the left in Fig. 2, and atmospheric pressure, the $(4 \times 4)$ phase is the most stable for the temperature range 350-530 K (incl. corrections, 460-640 K). For higher temperatures, up to about $720 \mathrm{~K}$ (incl. corrections, $830 \mathrm{~K}$ ), on-surface adsorbed oxygen is the only stable species. For temperatures beyond this, there are no stable species except for $\mathrm{O}$ atoms adsorbed at under ${ }^{3}-$ coordinated surface $\mathrm{Ag}$ atoms, such as next to a vacancy or step edge, which are stable to $865 \mathrm{~K}$ (incl. corrections, $975 \mathrm{~K})$. From the above analysis, our results point to oxygen atoms of the thin $(4 \times 4)$ surface-oxide structure as the main species actuating ethylene epoxidation as this occurs experimentally at $p_{\mathrm{O}_{2}}=1 \mathrm{~atm}$. and $T=500$ $600 \mathrm{~K}$, while either low coverages of on-surface oxygen or oxygen adsorbed at defects may play a role in the higher temperature catalytic reactions.

In Fig. 3, we consider just the lowest energy structures as a function of temperature $(T)$ and pressure $(p)$, yeilding the phase-diagram. Here the $p$ - $T$ range in which the various structures are stable can quickly be seen. We note that the phase-diagram is in-line with available wellestablished experimental results, namely, (i) that under UHV conditions, only very low coverage (0.03 ML) of chemisorbed $\mathrm{O}$ is stable up to about $490 \mathrm{~K}$ and (ii) that the $(4 \times 4)$ phase is stable at low temperatures (and UHV pressure) and that it decomposes at $580 \mathrm{~K}{ }^{22}$ Figure 3 furthermore shows that regardless of the pressure, the ordering of the phases is the same. Thus, the same phase should be observable at different pressures if the temperature is adjusted accordingly, which indicates a way to bridge the pressure gap - providing that formation of the structure is not prevented by kinetics.

It is interesting to consider the "removal" energy of a single (uppermost) $\mathrm{O}$ atom of the $(4 \times 4)$ structure, which is the energy required to move the $\mathrm{O}$ atom into the vacuum. This energy is the strongest that we found for the $\mathrm{O} / \mathrm{Ag}$ system, namely, $\sim 3.9 \mathrm{eV}$, which is due to the presence of the lower lying $\mathrm{O}$ atoms which stabilizes the upper $\mathrm{O}$ atoms. Compared to other $\mathrm{O} /$ metal systems, however, this does not constitute a strong bond. In fact, it represents an intermediate value, very similar to the identified "optimum" values for highest catalytic activity

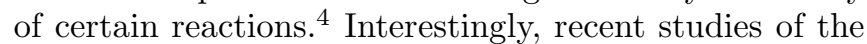
$\mathrm{CO}$ oxidation reaction over $\mathrm{Ru}$ have surprisingly found that oxides play an active role for reaction, in contrast to the hitherto believed pure metal. ${ }^{26,27}$ Ruthenium is a poor oxidation catalyst since it binds $\mathrm{O}$ too strongly, but at the high temperature and pressure conditions where $\mathrm{Ru}$-oxides form, a new weaker $\mathrm{O}$ species appears and the system is then catalytically very active. Thus it appears that the interaction of oxygen with transition metal catalysts can serve to "tune" the O-metal bondstrength for optimum reactivity through formation of various oxidelike species.

In conclusion, through DFT calculations, and including the effect of the environment, we obtained the pressure-temperature phase-diagram for $\mathrm{O} / \mathrm{Ag}(111)$. The results show that a proposed molecular ozone-like species adsorbed at a surface vacancy is energetically unfavorable, as is bulk-dissolved oxygen, which puts a question mark over the latter's hitherto thought important role in oxidation reactions. Our results also show that silver oxide and oxide-like films can also be ruled out as playing an important role due to their low thermal stability. On the other hand, the calculations reveal that silver 


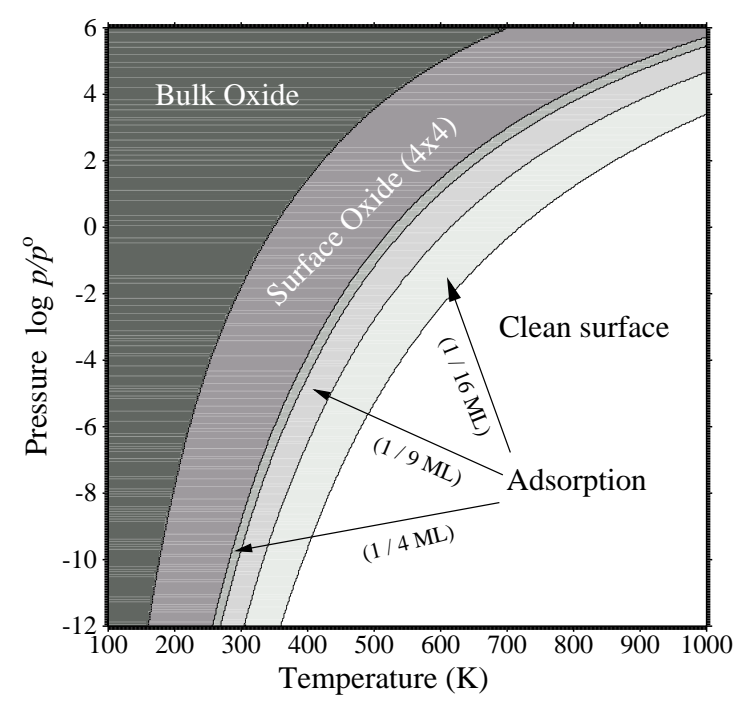

FIG. 3: Calculated $(p, T)$ phase-diagram for the oxygen$\mathrm{Ag}(111)$ system showing the stable structures.

can support a thin surface oxide-like structure under the temperature and pressure conditions of ethylene epoxidation and we propose that such atomic $\mathrm{O}$ species are actuating the reaction. For even higher temperatures, only very low concentrations of chemisorbed $\mathrm{O}$ are sta- ble, or $\mathrm{O}$ atoms adsorbed at under-coordinated surface Ag atoms. Therefore these species could possibly play a role in the catalytic oxidation reactions that take place at high temperature. We note that an understanding of a full catalytic cycle requires a kinetic modeling that includes all reactant species and intermediates, which, for complex systems such as those discussed here, is not yet possible. The present investigation represents a crucial first step towards this aim, which, using the approach of atomistic ab initio thermodynamics, identifies (and excludes) oxygen species that are present under the conditions of catalysis, and helps shed new light upon the function of silver as an oxidation catalyst.

More generally, our results highlight the formation and importance of various oxides under catalytic conditions, be it in weaker bonded $\mathrm{O} /$ noble-metal systems, such as $\mathrm{O} / \mathrm{Ag}$, or in stronger bonded $\mathrm{O} /$ transition-metal systems, as e.g., O/Ru. For transition metals that form bulk oxides with intermediate heats of formation, e.g. $\mathrm{PdO}$, under conditions typical of catalysis, we may expect that both bulk oxides and thiner surface-oxides could occur, depending sensitively on the pressure and temperature.

We thank D. King and R. Schlögl for helpful discussions.
1 Handbook on Heterogeneous Catalysis, Eds. G. Ertl, H. Knözinger and J. Weitkamp, (Wiley, New York, 1997) Vol.1.

2 A. A. Balandin, Adv. Catal. Rel. Subj. 19, 1 (1969).

3 R. A. van Santen and J. W. Niemantsverdriet, Chemical Kinetics and Catalysis (Plenum Press, New York, 1995).

4 J. K. Nørskov, et al., and references therein, J. Catal. 209, 275 (2002).

5 R. R. Chianelli, et al., Appl. Catal. A 227,83 (2002).

6 A. Nagy, et al. J. Catal. 182, 417 (1999); ibid J. Catal. 188, 58 (1999); Appl. Catal. A 188, 337 (1999).

7 D. Herein et al., Z. Phys. Chem. 197, 67 (1996).

${ }^{8}$ R. A. van Santen and H. P. C. E. Kuipers, Adv. Catal. 35, 265 (1987).

9 A. I. Boronin, et al. Kin. Catal. 40, 721 (1999).

10 V. I. Bukhtiyarov, et al. Catal. Lett. 74, 121 (2001).

11 X. Bao et al. Phys. Rev. B 54, 2249 (1996).

12 G. C. Bond and D. T. Thompson, Catal. Rev. Sci. Eng. 41, 319 (1999), and references therein.

13 Y. Uchida, et al., Surf. Sci. 401, 469 (1998).

14 J. Chevrier, et al., Surf. Sci. 355, 1 (1996).

15 Th. Schedel-Niedrig et al., Phys. Chem. Chem. Phys. 2, 3473 (2000).

16 N. Troullier and J. L. Martins, Phys. Rev. B 43, 1993 (1991).
17 M. Fuchs and M. Scheffler, Comput. Phys. Commun. 116, 1 (1999).

18 M. Bockstedte et al. Comput. Phys. Commun. 107, 187 (1997).

19 J. P. Perdew, K. Burke, and M. Ernzerhof, Phys. Rev. Lett. 77, 3865 (1996).

${ }^{20}$ W. X. Li, C. Stampfl, and M. Scheffler, Phys. Rev. B 65, 075407 (2002); Phys. Rev. B 67, 045408 (2003).

21 C. I. Carlisle et al., Phys. Rev. Lett. 84, 3899 (2000); Surf. Sci. 470, 15 (2000).

${ }^{22}$ W. X. Li, C. Stampfl, and M. Scheffler, to be published.

23 CRC Handbook of Chemistry and Physics, edited by R. C. Weast, 55 ed. Cleveland, Ohio, 1974-1975.

${ }^{24}$ K. Reuter and M. Scheffler, Phys. Rev. B 65, 035406 (2002)

25 We compared results obtained using the GGA and LDA for two selected structures i.e. on-surface $\mathrm{O}$ with coverage $1 / 4 \mathrm{ML}$ and the $0.50 \mathrm{ML}$ oxide-like structure. Although the absolute value of the free energy is 14.6 and $14.3 \mathrm{meV} / \AA^{2}$ larger respectively within the LDA, the phase boundary position differs by only $0.021 \mathrm{eV}$ suggesting that the whole phase diagram is very similar within LDA and GGA.

26 H. Over et al., Science 287, 1474 (2000).

27 C. Stampfl et al., Surf. Sci. 500, 368 (2002). 